\title{
The molecular receptive range of an odorant receptor
}

\author{
Ricardo C. Araneda, Abhay D. Kini and Stuart Firestein
}

Department of Biological Sciences, Columbia University, New York, New York 10027, USA

Correspondence should be addressed to S.F. (sjf24@columbia.edu)

\begin{abstract}
An odor perception is the brain's interpretation of the activation pattern of many peripheral sensory neurons that are differentially sensitive to a wide variety of odors. The sensitivity of these neurons is determined by which of the thousand or so odor receptor proteins they express on their surface. Understanding the odor code thus requires mapping the receptive range of odorant receptors. We have adopted a pharmacological approach that uses a large and diverse pool of odorous compounds to characterize the molecular receptive field of an odor receptor. We found a high specificity for certain molecular features, but high tolerance for others-a strategy that enables the olfactory apparatus to be both highly discriminating, and able to recognize several thousand odorous compounds.
\end{abstract}

Sensory system description and analysis have traditionally used the notion of a 'receptive field,' which is defined as the stimulus range that can be detected at any particular stage of processing. In the visual, auditory and somatosensory systems, this characterization has led to important advances in understanding how a perception is constructed from the raw sensory stimuli. In these systems, the physical energy of the stimulus is converted into a topographical map in the brain, such that stimuli can be parsed in various combinations to form novel perceptions. However, the olfactory system presents a different sort of problem to the brain. The vertebrate nose can detect and discriminate among thousands of low-molecular-weight organic compounds that possess an enormous diversity of chemical structures and properties ${ }^{1}$. Unlike other sensory stimuli, however, there are no primaries (as in overlapping wavelengths of light for colors) or fundamentals (as in harmonic frequencies in sound waves) into which complex stimuli can be resolved. How the brain maps this diverse stimulus set onto neural space remains a puzzle. The first step toward comprehending this process in olfaction would be to derive a 'molecular receptive range' for the primary sensory apparatus ${ }^{2}$.

The cloning of a large family of G-protein-coupled receptors (GPCRs) in the nose provided the first molecular basis for understanding olfactory coding ${ }^{3}$. Some 1000 odor receptors (ORs) are expressed by the olfactory sensory neurons (OSNs) lining the nasal epithelium, making this by far the largest single family of GPCRs. Each of the receptors may additionally possess multiple binding sites, adding up to a large number of possible receptor-ligand interactions. An important organizational principle that emerged from gene targeting studies was that the axons of all neurons expressing the same receptor converge onto the same glomerular target in the olfactory bulb ${ }^{4}$. Thus, the receptive field of a glomerulus is equivalent to the molecular receptive range of the odor receptor expressed by its innervating neurons.

From a growing body of psychophysical, physiological and biochemical investigations, it seems that many odors are recognized by more than one receptor, and most receptors probably recognize multiple odors ${ }^{2,5-9}$. However, the correlation between odors and receptors is known for only a few receptors and is generally based on relatively small stimulus sets ${ }^{5,6,10-12}$. A complete description of the binding characteristics for even a single odor receptor is not currently available. This is in contrast to many other members of the GPCR superfamily, where pharmacological strategies have provided a profile of agonists and agonist characteristics (that is, the pharmacophore), as well as antagonists, which may be thought of as defining the receptive range of a receptor. Here we have adapted an approach rooted in medicinal chemistry to define the molecular receptive range of a particular OR, the I7 receptor ${ }^{13,14}$. This receptor, the first OR to be paired with its cognate ligand, had previously been tested with a wide ranging, but essentially random, panel of odor ligands 5 . Although this testing was enough to identify a short-chain aliphatic aldehyde, octanal, as a primary agonist, the questions of specificity, molecular range and tuning of this or any odor receptor remain largely uninvestigated. Using a somewhat more rational chemical approach, we tested the activity of 90 molecules from a panel of 200 screened odors, including aromatic and aliphatic short-chain compounds with alternate functional groups, and varying degrees of saturation and substitution along the backbone. From these diverse compounds, we found several features that predict activity or inactivity at the I7 receptor. Thus, we defined a detailed molecular receptive range for this receptor, and by extension, for the primary sensory neurons in which it is expressed.

\section{RESULTS}

Expression of the recombinant rat I7 odor receptor gene can be induced in rat olfactory epithelia with an engineered adenovirus vector ${ }^{15}$. Upon infection, I7 receptor expression is driven in many sensory neurons. These neurons can be recognized by the presence of a green fluorescent protein (GFP) marker, whose gene is also contained in the adenoviral vector. Electroolfactogram (EOG) recordings from these sensory neurons show increased responses to a series of activating ligands (odors), compared to controls ${ }^{16,17}$. This electrophysiological response is a reliable indicator of the stimulus-induced activity of OSNs, and can therefore be used to measure the differential response of neurons expressing the I7 receptor to a varied set of ligands ${ }^{5,10}$. 


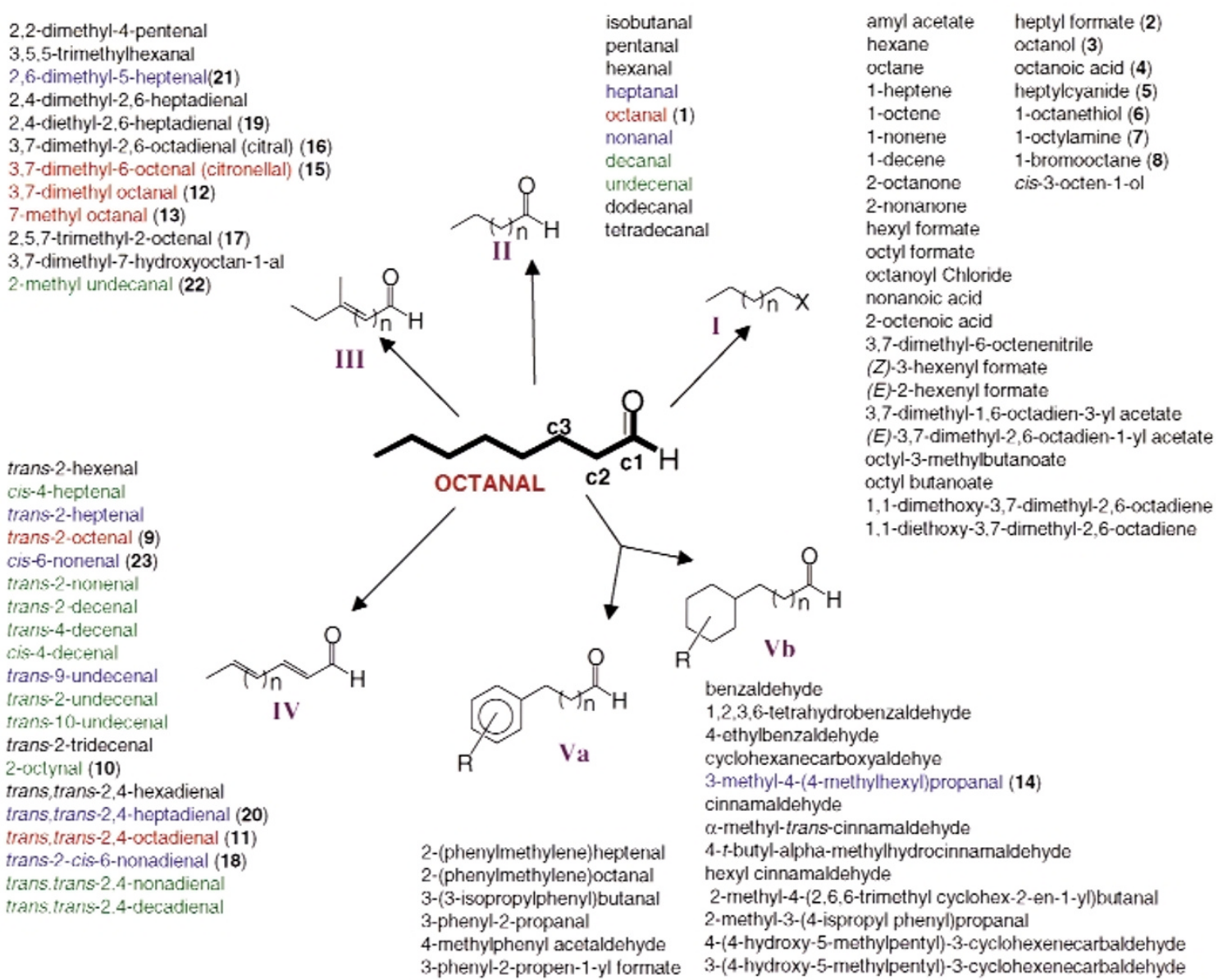

Fig. 1. Strategy used for the selection of potential agonists of O R-17. U sing octyl aldehyde as a template, potential ago nists were selected according to the number of carbons, degree of saturation, branching and substitutions. Compounds are grouped in five categories corresponding to substitutions of the aldehyde group (I), n-aldehydes (II), n- and branched singly unsaturated aldehydes (III), singly and doubly unsaturated n-aldehydes (IV) and cyclic and aromatic aldehydes ( $\mathrm{Va}$ and $\mathrm{Vb})$. C ompounds that showed a significant activity are color coded to illustrate the values of infected minus control, that is, the fold increase in response relative to a normal animal (see Methods). The highest value, 1.52, was for trans,trans-2,4-octadienal and the lowest, 0.0398, for 2-methyl-undecanal. Red, 1.5-0.92 (highest); blue, 0.91-0.41 (medium); green, 0.40-0.04 (lowest).

By this method, we previously established that the I7 odor receptor is activated by saturated aliphatic aldehydes with backbone chains of 7 to 10 carbons, centered on octyl aldehyde (or octanal) $)^{5}$. We therefore used octyl aldehyde (1) as a template for deriving potential ligands (Fig. 1). Many of these compounds are commercially available; others are not generally available but have been developed in the fragrance industry and were provided for this study. To explore the molecular range of the receptor, we tested 90 compounds, chosen to represent alterations in functional group (I), backbone chain length (II), degree of unsaturation (III, IV) and side chain substitution (III, V; Fig. 1). Although the majority of these compounds showed no activity at the I7 receptor, consistent with the notion that these receptors are capable of a high degree of discrimination, several compounds with potentially informative structures were active; they are highlighted by green, red and blue coloring (extent of increase in response, red $>$ blue $>$ green). In many cases, compounds that were very closely related by chemical structure did not share similar activity. Nevertheless, differences between active and inactive compounds revealed several important determinants for I7 receptor agonists.

\section{The carbonyl aldehyde is critical}

The initial target of chemical modifications was the aldehyde functional group. In Fig. 2, the aldehyde moiety of octanal has been replaced with other functional groups, including formate (2), hydroxyl (3), carboxylate (4), nitrile (5), thiol (6), amine (7) and halide (8) groups. Although each of these alternate functional groups leaves the molecular shape approximately unchanged, and provides some substitute for either the hydrogen bond acceptor function or the partial charge of the carbonyl group, the functional groups were nonetheless ineffective in activating the receptor. Compounds 3, 4 and 5 were tested at 10 times the concentration generally used in our studies $(1 \mathrm{mM})$, and still failed to show any activity. Eight carbon ketones and esters were also inactive (data not shown). Other compounds with these functional groups and different carbon chain lengths also did not generate activity (data not shown). Even the formates, the only class of molecules that maintain an aldehyde-like structure at the $\mathrm{C} 1$ carbon (with the $\alpha$-methylene group changed to an oxygen; Group I, Fig. 1), showed no activity. These data demonstrate the absolute requirement of the aldehydic group for activity at the I7 receptor. However, the aldehyde carbonyl was not, by itself, sufficient for activity, as several other aldehyde-containing com- 
a<smiles>[13CH3][13CH2][13CH3]</smiles>

Hexyl formate (2)<smiles>CC(C)(C)CC=O</smiles>

Octanal (1)
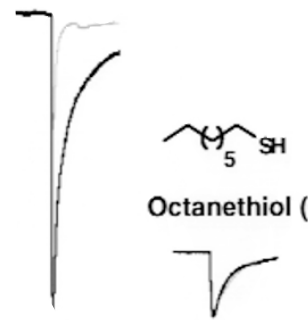

b

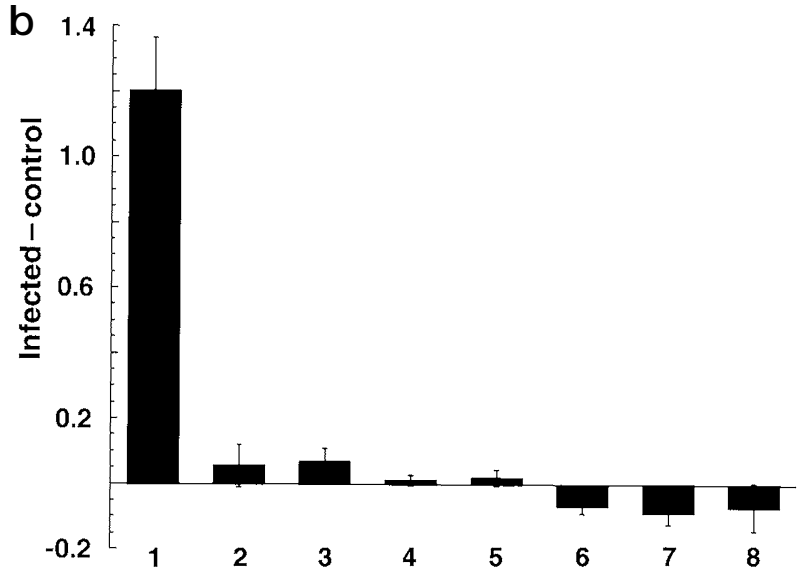

pounds were inactive. For example, many aromatic aldehydes (such as benzaldehyde), and other substituted aliphatic aldehydes (see below) were not agonists.

\section{Importance of molecular length}

The restricted range of active saturated aldehydes (C7-C11) suggests that the length of the molecules might also be important. Whereas the saturated short-chain aldehydes were predicted to be in the extended form, they could in fact rotate around any of the carbon bonds, and once bound to the receptor, they could energetically assume a variety of shapes. However, trans unsaturated compounds of similar backbone composition are more likely to be restricted to the extended conformation (for example, see compounds 9,11 and 20) because rotation around double bonds is restricted. Figure 3 a shows responses to 12 singly and doubly unsaturated compounds that were all active at the I7 receptor (with the exception of trans, trans-2,4-hexadienal). Both cis and trans double bonds were well tolerated. Activity produced by the unsaturated compounds showed the same restriction in carbon number as was previously observed in the saturated compounds-only molecules with 7-11 carbons were active.

To calculate molecular length, we chose only those trans unsaturated compounds that were predicted by molecular modeling to be in the extended configuration. This allowed us to use these compounds as a kind of ruler, to estimate the acceptable range

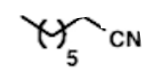

Heptyl cyanide (5)
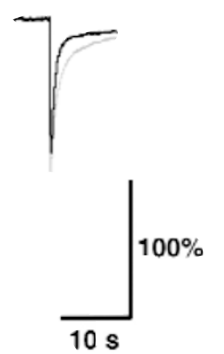

Fig. 2. Replacing the aldehyde group reduces activity. (a) Representative EOG recordings from control (gray) and infected tissue (black). All EO $G$ responses are normalized to the reference odorant, amyl acetate (AA), which is given the value of 1 (see Methods). (b) Comparison of the subtracted average control from Ad-I7 infected for 8-carbon (C 8) molecules with different functional groups. Of all compounds in this class, only the responses to octanal are significantly increased in infected animals. All compounds were tested at $10^{-3} \mathrm{M}$ except octanol, octanoic acid and heptyl cyanide, which were tested at $10^{-2} \mathrm{M}$ as well (similar results were obtained at $10^{-3} \mathrm{M}$ ). The bars correspond to the subtraction of the average control value from the average Ad-17-infected responses obtained from EO G recordings as shown in (a).

of sizes for an active molecule. When the calculated length of the molecule in the extended conformation was plotted against activity, all the active molecules were shown to have lengths from 8 to $12 \AA$ (Fig. 3b).

\section{Effects of substitutions along the carbon backbone}

Because octanal (1) seemed to be the most potent agonist, we chose to explore the effect of various modifications along the octanal backbone, to gain further insight into receptor-ligand interactions. For these experiments, we analyzed the effect of unsaturation alone or in combination with added methyl groups and other substituents (9-17). Seven of the nine compounds shown in Fig. 4a (9-15) were active ligands for the I7 receptor, generating responses from 1.5 to more than 3 times greater than responses in the control tissue, suggesting a wide tolerance for variation along the carbon backbone. Only 2,5,7-trimethyl-2octenal (17) failed to show activity, whereas citral (16) produced only a small increase in activity, and only when delivered at a high concentration $(10 \mathrm{mM})$. Citral is a mixture of 2 isomers known as geranial $(Z)$ and neral $(E)$ in the ratio of 3:2 $(Z: E)$. Each of the isomers was also tested individually and found to have nearly identical activity to the mixture (data not shown). All of these compounds were tested at a 10 -fold lower concentration, and the same profile of activities was observed (data not shown).

The lack of activity in compounds 16 and 17, in spite of their structural similarity to several of the agonists, suggested that they could block the ability of octanal to activate the I7 receptor. To test this hypothesis, we measured the response to octanal alone or in the presence of citral (16) and 2,5,7-trimethyl-2-octenal (17) using calcium imaging of single infected neurons (Fig. 4b). This protocol allowed us to do competition experiments under conditions more controlled for compound delivery. Individual infected neurons responded to octanal at concentrations as low as $0.3 \mu \mathrm{M}$ (data not shown) and gave a nearly saturating response when applied at 10-30 $\mu \mathrm{M}$. Citral presented at concentrations from 0.1 to $1 \mathrm{mM}$ gave no response. However, the co-application of citral $(100 \mu \mathrm{M})$ and octanal $(10 \mu \mathrm{M})$ reduced the response to octanal by $39.4 \pm 4.9 \%(n=4)$ as compared to control. Although these data alone do not allow us to identify whether this is a case of antagonism or partial agonism, we believe that because citral seemed to produce a small response in the EOG recordings, which measure the summed response of many neurons, it is likely that citral is acting as a partial agonist. Compound 17, which, similarly to citral, failed to activate individual neurons when 


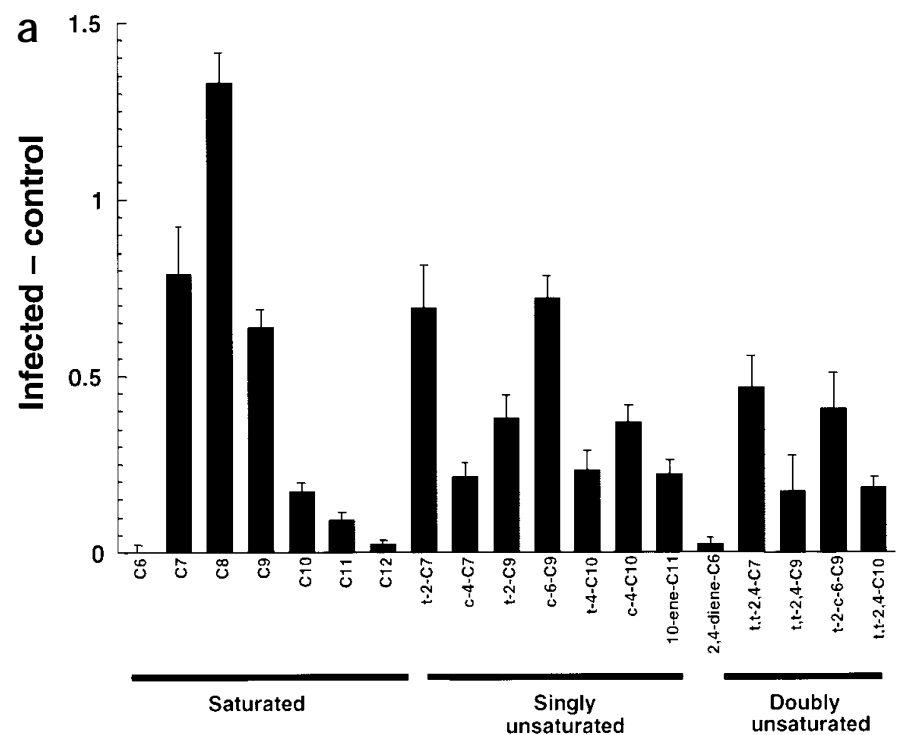

b

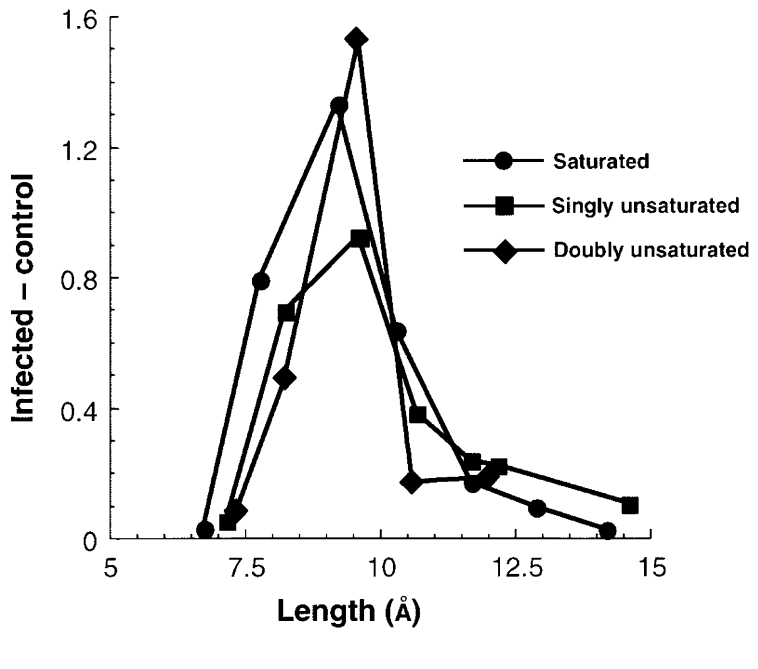

Fig. 3. Length of the molecule is important for activity. (a) Comparison of the subtracted average control from Ad-17 infected animals for saturated and unsaturated aldehydes. Saturated aldehydes in the C 7-C 11 range showed significantly increased responses in infected animals. In the same range, unsaturated aldehydes with one (I) or two (II) double bonds also showed increased responses. All compounds were tested at $10^{-3} \mathrm{M} . \mathrm{C}(\mathrm{x})$ refers to the number of carbons in the aldehyde backbone; $t$, trans and c, cis. The statistical significance of the data was evaluated with the unpaired t-test; in this case $p<0.001$ for all active compounds. The responses to 2,4-hexadienal and the $\mathrm{C} 12$ aldehyde were not significantly different from control ( $n=8-50$ animals; error bars represent s.e.m.). (b) Subtraction of control from infected versus length for some of the $\mathrm{C} 6-\mathrm{C} 12$ saturated and unsaturated aldehydes tested. The aldehyde length was calculated for the predicted lowest energy conformer using Macromodel v6.5 softwar $\mathrm{e}^{28}$ and was measured from the carbonyl group to the last carbon, assuming an extended conformation. The molecular length of all molecules was calculated using atom center distances. $\bullet$, saturated; $\boldsymbol{\square}$, singly unsaturated; $\bullet$, doubly unsaturated.

observed with calcium imaging, had no antagonistic effects on the response to octanal.

A more detailed analysis of the structures in Fig. 4a could provide valuable steric information regarding the key features governing binding. Comparison of the predicted low-energy conformations of active molecules (Fig. 5) showed that though they share a common configuration close to the carbonyl group, they vary extensively toward the tail region. In the active compounds, added methyl groups and double bonds located after the $\mathrm{C} 4$ carbon seemed to have little effect on the ability of the ligand to activate the receptor. For example, molecules such as trans-2-cis-6-nonadienal (18), which due to the cis double bond at C6 exist primarily in a non-extended form, are nonetheless active. Even a cyclohexyl ring at C4 (14) did not interfere with activity.

Substituents in the C2 or C3 positions, however, were not similarly tolerated (Fig. 5b). Citronellal, with a methyl group at C3, but no double bond, was active. Trans-2 octenal, with a double bond at C2 but no methyl group, was also active, as was 2, 6-dimethyl-5-heptenal (21) with a methyl group, but no double bond, at C2. However, citral (16), with a double bond at C2 and a methyl group at C3 showed only slight activity (and only at high concentrations), whereas the fully saturated version of citral, tetrahydrocitral (12), with identical methyl group substitutions but no double bonds, was highly active. Likewise, 2,4-dimethyl2,6-heptadienal (19) and 17, both with double bonds and methyl groups at C2, were inactive. Thus, a double bond or a methyl group in close proximity to the carbonyl was tolerated, but the combination of the two was not.

These observations were consistent with structural constraints near the functional group of these molecules. Although the inactive compounds shown in Fig. 5b have many striking structural differences in the tail region (such as kinks and bent conformations from $\mathrm{C} 4$ back), most of these differences are shared with other compounds that were active, suggesting that the differences are not the critical determinants of activity. Rather, the low activity observed for 16, and the inactivity of 17 and 19 , can be attributed to the $\mathrm{sp}^{2}$ geometry at carbons $\mathrm{C} 2$ and $\mathrm{C} 3$, which forces the methyl group into the plane of the carbonyl and the $\alpha, \beta$-double bond $(-\mathrm{C} 2=\mathrm{C} 3-)$. Without the double bond (that is, in the $\mathrm{sp}^{3}$ state; see compounds 12,15 ) the methyl groups assume a 'gauche' spatial relationship to the carbonyl (Fig. 5c). This relationship is evident in the overlay of the predicted lowest energy conformers $(9,14,15,17,18$; Fig. 5b) and in the Newman projections (Fig. 5c). The constraint of having a methyl group at $\mathrm{C} 2$ or $\mathrm{C} 3$ in conjunction with a double bond seems to be enough to render the compounds 17 and 19 inactive, suggesting a strong steric constraint in the vicinity of the aldehyde moiety.

\section{Discussion}

It is generally accepted, although not definitively established, that most individual olfactory sensory neurons express only one of the approximately 1000 odor receptor genes ${ }^{18}$. Additionally, it seems that all neurons expressing a particular OR project their axons to one of two glomerular targets in the olfactory bulb ${ }^{4}$. The glomerulus, in turn, is innervated by some 25 second-order cortical-like neurons known as mitral cells ${ }^{19}$. Therefore, the primary receptive field of the mitral cell, a higher-level processing neuron, is, in large part, determined by the molecular receptive range of the ORs. Characterizing this receptive range is then a prerequisite for understanding the formation of an olfactory percept.

Using concepts of rational drug design, we have concentrated 
a

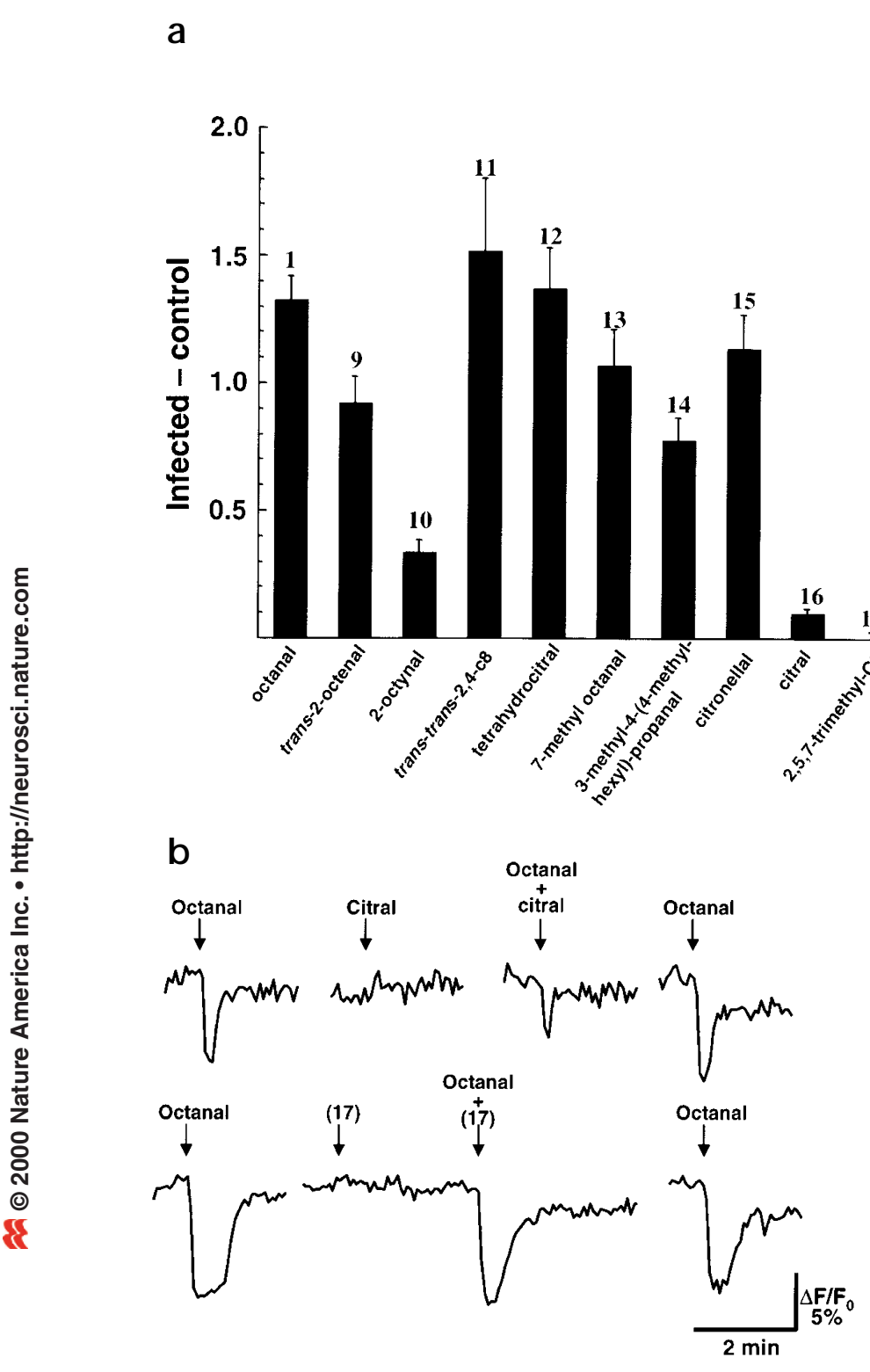

on analyzing the activity of a group of short-chain alkyl aldehydes from an initial panel of nearly 200 screened odorous compounds, all at the I7 odor receptor. These aldehydes were chosen to provide a range of structural variations that would reveal the key features of an activating ligand. In parallel with medicinal chemistry, where receptor tuning is described in terms of pharmacophores, we find common traits in the activating aldehydes ('aromaphores'). Such rigorous investigation with many chemically related ligands, as done here, has enabled us to produce a tuning profile for the I7 odor receptor: highly specific for an aldehyde group, with strict steric prerequisites around the carbonyl, but rather indiscriminate for structural variations at the tail of the molecule.

The unavailability of a reliable expression system for olfactory receptors has made the characterization of their pharmacological profile a difficult task. Moreover, in the few receptors studied so far, only a small number of molecules have been used to determine their specificity ${ }^{6,10}$. Response patterns from physiological and biochemical studies suggest receptive ranges that

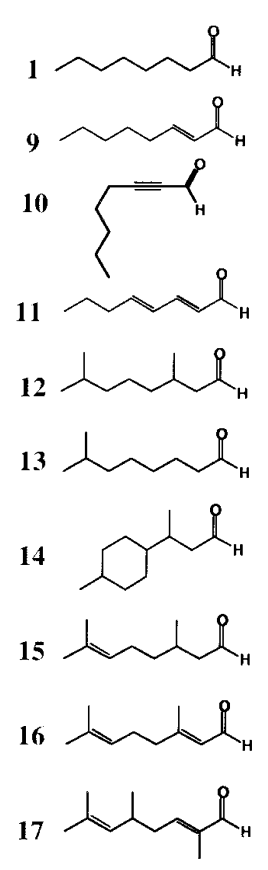

Fig. 4. Effect of substitution and unsaturation on the activity of $\mathrm{C} 8$ aldehydes. (a) Comparison of the subtracted control from Ad-17 infected for analogs of octanal. All compounds are shown at $10^{-3} \mathrm{M}$ except for citral $\left(10^{-2} \mathrm{M}\right)$. Citral, only at this high concentration, produced and small but significant increased response $(p<0.001)$. At all concentrations tested, 2,5,7-trimethyl-2-octenal did not produce a significant increase in responses in infected animals. All other C 8 analogs had increased responses $(p<0.001)$ at $10^{-3}$ M. (b) Citral reduced octanal responses, as shown in $\mathrm{Ca}^{2+}$ imaging, in isolated olfactory neurons expressing 17 receptors. 17-expressing cells were recognized by the presence of GFP. $O$ ctanal ( $10 \mu \mathrm{M}$ and $30 \mu \mathrm{M}$, top and bottom panel respectively) produced an increase in $\mathrm{Ca}^{2+}$ as shown by the change in the emitted light. In the presence of citral $(100 \mu \mathrm{M})$, top, but not in the presence of 2,5,5-trimethyl-2-octenal (100 $\mu \mathrm{M})$, bottom, the response to octanal is reduced.

possess both breadth and specificity. To account for this, it has been proposed that most ORs respond to multiple odors that, although different in many respects, must share certain molecular determinants ${ }^{20,21}$. A particularly notable feature we found in the I7 receptor was the absolute requirement for an aldehyde carbonyl. Substitution with any other functional group removed any agonist activity, even when higher concentrations were used. An absolute requirement for an aldehyde group suggests that the ligand-receptor interaction site requires a specificity greater than an overall negative charge, or the hydrogen bonding capability at one end of the molecule, as many of the carbonyl substitutions we used fulfilled these criteria (see Group I, Fig. 1). The importance of the aldehyde carbonyl has also been suggested in modeling studies, which have provided evidence that the carbonyl may form electrostatic bonds with lysine 164 within the I7 binding pocket ${ }^{22}$. This close correspondence suggests that future studies using mutations and modeling, as in rational drug design, should be helpful for understanding olfactory receptors and their ligands ${ }^{22,23}$.

Another important determinant of activity was molecular length. Molecules shorter than $8 \AA$, and with fewer than seven carbons in the backbone, were not active, even though all of the compounds tested had strong odor qualities and exhibited large EOG responses (that is, they were able to bind to some receptor). Molecules longer than $12 \AA$, or with more than 11 carbons, were also poor agonists, although they too are able to evoke distinct odor qualities. The relationship of length to activity has also been observed in mitral cell recordings, further suggesting that this property of the molecules is relayed to higher centers ${ }^{2}$. The relationship between activity and length observed here suggests some molecular constraints imposed by the dimension of the binding site. Longer molecules simply may not fit into the restricted binding region. Shorter molecules that are inactive either may partially occupy the binding site and fail to activate the receptor, or simply may not be able to reside stably in the binding site at all. This issue may be eventually be resolved with 

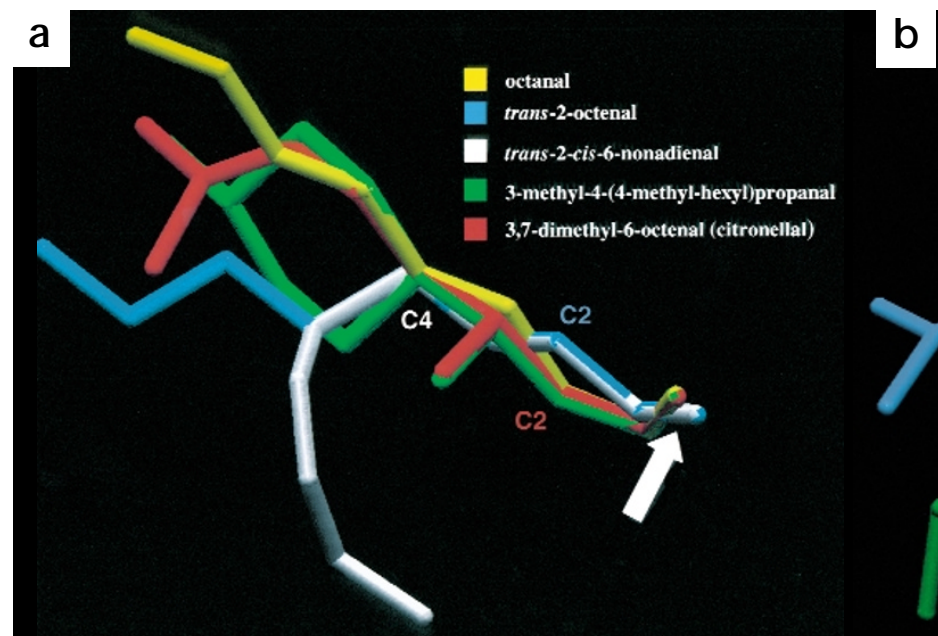

b 2,4 -dimethyl-2,6-heptadienal 3,7-dimethyl-6-octenal (citronellal) 3,7-dimethyl-2,6-octadienal (citral) 2,5,7-trimethyl-2-octenal 3-methyl-4-(4-methyl-hexyl)propanal 4

Fig 5. 0 verlay of the predicted lowest energy conformers of long-chain aldehydes. Energy minimizations and conformational searches (Monte Carlo method) were conducted using the MM2* force field in Macromodel v6.5. The number of conformers found within $10 \mathrm{~kJ} / \mathrm{mole}$ for each molecule, while conducting the conformational search for each molecule, are in Methods. The molecules were superimposed in Macromodel, using, in all cases, identi-
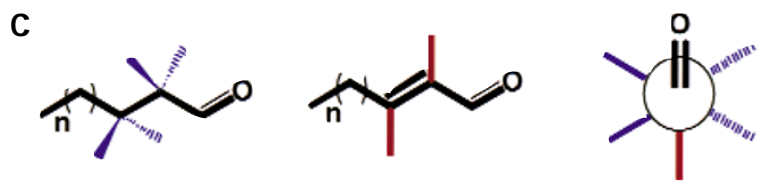

4 were overlaid using a rigid body fit. (a) 0 verlay of five aldehydes that are capable of activating the receptor: octanal (yellow), trans-2-octenal (blue), trans-2-cis-6-nonadenial (white), 3-methyl-4-(4-methylhexyl)-propanal (green), and citronellal (red). (b) 0 verlay of active (citronellal, blue) and inactive aldehyde molecules (2,4-dimethyl-2,6-heptadienal, red; citral, E isomer, neral, most abundant in mixture, white; 2,5,7-trimethyl-2-octenal, green). (c) G raphical representation of the effect of $\mathrm{sp}^{3}$ (left) and $\mathrm{sp}^{2}$ (center) hybridization on methyl groups at the $\mathrm{C} 2$ and $\mathrm{C} 3$ carbon atoms. N ew man projection (right) shows the effect of a double bond at $\mathrm{C} 2$ on methyl groups at $\mathrm{C} 2$ and $\mathrm{C} 3$. The double bond forces the methyl groups into the same plane as the carbonyl and the $\alpha, \beta$-double bond, whereas without the double bond, the methyl groups are free to rotate out of the plane of the carbonyl.

competition experiments. Whereas the differences in solubility and desolvation enthalpy, which are a function of increased carbon number, may have some confounding effect on this relationship, we note that the rapid decline in acceptable molecular length is consistent with the notion that repulsive forces at longer molecular lengths may become important.

Within the acceptable length range for active molecules, structural variations toward the tail end were more tolerated. This suggests that the interaction at this region of the molecule, as opposed to the carbonyl head, involves less specificity. This might indicate the participation of a flexible hydrophobic binding pocket, where, provided that the length of the ligand is correct, the tail could assume various conformations. Indeed, many of the active compounds were saturated aliphatics and therefore energetically free to adopt a large number of conformational states. If one thinks of all the possible conformers that could be occupied by the tails of the active molecules as occupying an idealized spherical volume, then the minimum diameter of this sphere would be the width of the cyclohexyl ring (including the hydrogen atoms) from the active compound 14, and the maximum diameter would be the longest distance from the $\mathrm{C} 4$ carbon to the terminal carbon (in this case, the $\mathrm{C} 10$ carbon of the mildly active compound decanal). These values, 4.2 and $7.1 \AA$ respectively, could roughly define a hydrophobic space in the binding pocket that might accommodate this portion of the molecule.

In contrast to the flexible nature of the interactions at the tail end of the molecule, there are very prominent structural constraints in the immediate environment around the aldehyde group. The presence of a double bond at C2-C3 (for example, compound 9 and Fig 1, group IV) or a methylation at C2 (12, $15,18,21)$ does not significantly diminish activity. However, the combination of a double bond and a methyl group dramatically reduces $(16)$ or completely abolishes activity $(17,19)$. In these compounds $(16,17,19)$, the $\mathrm{sp}^{2}$ hybridization at $\mathrm{C} 2 / \mathrm{C} 3$ forces the methyl group into the plane of the carbonyl and the $\alpha, \beta$-double bond. It could be argued that the low activity exhibited by citral is due to its bent predicted lowest energy conformation (Fig. 5b). However the similarly bent trans-2-cis-6-nonadienal was active (Fig. 5a). For trans-2-cis-6-nonadienal, there exist at least 15 conformers in an extended conformation within a $5 \mathrm{~kJ}$ range that the receptor can access. These conformers are all based on the rotation of the C4-C5 dihedral angle. Similarly, extended conformations that the receptor could access also exist for citral. Therefore, the observed loss in activity in these compounds (Fig. $5 \mathbf{b}$ ) is probably due to the apparently rigid steric requirement at the head of the binding pocket, which seemingly cannot tolerate any substitutions at $\mathrm{C} 2$ or $\mathrm{C} 3$ in the plane of the carbonyl. This situation is reversed for $12,15,18$ and 21 , which are methylated at $\mathrm{C} 2$ or $\mathrm{C} 3$ but, because of the $\mathrm{sp}^{3}$ hybridization on these carbon atoms, they are not forced into the plane of the aldehyde carbonyl.

Of particular interest in this regard is the difference between citral (16) and 2,5,7-tri-methyl octenal (17). Neither was able to activate the receptor, but for apparently different reasons. Citral must have bound to the receptor, because it produced a small response in the EOG, although it must have bound only at high concentrations. Therefore, we reason that it was able to act as a partial antagonist, competing with octanal for a binding site. On the other hand, compound $\mathbf{1 7}$ had no antagonistic activity in the presence of octanal, and presumably failed to bind the receptor at all. Thus, citral bound, but had only low efficacy, whereas 17 had low binding ability. By our model, the important difference 
between the molecules is the position of the methyl group at C3 (16) versus C2 (17). Although we model citral as having its methyl group in the anti position $\left(180^{\circ}\right.$ from the aldehyde carbonyl), it actually exists in two isomers, neral and geranial ( $E$ and $Z$, respectively), and both have equivalent antagonistic qualities. Therefore, it was not the anti versus eclipsed orientation of the methyl group that was critical, but rather the methyl group's proximity to the carbonyl group. Differential effects of similar compounds, as seen in this case by the actions of 16 and 17, could provide the foundation for a pharmacologically based classification of odor receptors. Additionally, antagonism between odors at the receptor level could be involved in the perceptual complexities seen in odor mixtures.

Our data suggest that the I7 receptor is highly specific for an aldehyde group, has strict steric prerequisites around the carbonyl carbon, but is rather indiscriminate regarding structural variations at the tail of the molecule. Other receptors might be tolerant of different functional groups ${ }^{2,6}$, but very specific for molecular length or other structural components. This feature of localized molecular specificities would allow higher-order centers, integrating input from multiple OSNs each with specific molecular sensitivities, to make subtle discriminations between closely related odor compounds, while still allowing the primary receptors to participate in the perception of a potentially wide variety of odors.

Descriptions of receptor-ligand interactions can be approached by a variety of techniques, including receptor mutagenesis, pharmacology, medicinal chemistry and computational modeling. Given the difficulty of efficiently expressing ORs in heterologous systems, we used diverse chemical ligands to explore the nature of the binding process, and to define a receptor tuning curve. In concert with recent modeling efforts on GPCRs in general, and the ORs in particular, we can begin to appreciate how the olfactory system produces perceptions by extracting particular molecular features from a diverse chemical world.

\section{Methods}

All experiments followed NIH guidelines and were performed in compliance with the Columbia University Institutional Animal Care and Use Committee.

Construction of the Adex-I7-IRES-GFP adenovirus. The adenoviral vector AdexCAG-I7-IRES-GFP (Ad-I7) was the same as in previous work ${ }^{5}$. Briefly, the I7-IRES-GFP sequence was subcloned into the SwaI site of the cosmid vector pAdexlpCAw to create the cosmid vector pAdexI7IRES-GFP ${ }^{24,25}$. The pAdexlpCAw cosmid was created from the human adenovirus type 5 genome from which the E1a, E1b and E3 regions were deleted and replaced with an expression unit containing the CAG promoter (composed of the cytomegalovirus enhancer plus the chicken betaactin promoter ${ }^{26}$, a SwaI site and the rabbit beta-globin polyadenylation signal). The cosmid vector pAdexI7-IRES-GFP and the EcoT22I-digested DNA-terminal protein complex (DNA-TPC) of Ad5-dlX, which is a human type 5 adenovirus lacking the $\mathrm{E} 3$ region, were co-transfected into HEK293 cells by calcium phosphate precipitation. The recombinant adenovirus AdexCAG-I7-IRES-GFP was then generated by homologous recombination in the HEK293 cells. The same cells were used to propagate the recombinant adenovirus and then purified by cesium gradient centrifugation ${ }^{25}$. The viral titer was determined by plaque forming assay on HEK 293 cells.

Adenovirus infection of olfactory epithelia. Rats were infected as before ${ }^{5}$. Briefly, anesthetized animals were infected with $30-50 \mu \mathrm{l}$ of a rat Ringer solution containing the I7-GFP virus and $0.3 \%$ fast green dye. The solution was slowly injected through the nostril, into the right side of the nasal cavity, with a thin plastic tubing. The solution was allowed to remain in the nasal cavity. After recovery, the animals were maintained at room temperature with no other treatment until they were killed. The day of the recording, the animal was overdosed with anesthetics (ketamine and xylazine) and decapitated. The head was cut open sagitally, and the septum was removed to expose the medial surface of the olfactory turbinates. The right half of the head was mounted in a wax dish filled with rat Ringer. The medial surface of turbinates was face up and exposed to the air. A continuous stream of humidified clean air was gently blown on the turbinates through tubing to prevent tissue from drying. The opening of the tubing was $8 \mathrm{~mm}$ in diameter and placed approximately $10 \mathrm{~mm}$ from the turbinate surface.

EOG recordings. The EOG recording electrode was an $\mathrm{Ag} / \mathrm{AgCl}$ wire in a capillary glass pipette filled with rat Ringer solution containing $0.6 \%$ agarose. The electrode resistance was between 0.5 and $1 \mathrm{M} \Omega$. The recording pipette was placed on the surface of the olfactory epithelium and connected to a differential amplifier (DP-301, Warner Instruments, Hamden, Connecticut). Placement of the electrode was determined by visualizing GFP fluorescence with a modified stereomicroscope (Nikon, Tokyo, Japan). The EOG potential was acquired using an IT16 interface and an acquisition routine written with Igor Pro software (WaveMetrics, Lake Oswego, Oregon). Each odor was tested in at least three different infected animals, and for most experiments, two electrodes and two amplifiers were used to record EOGs from two different sites of epithelium simultaneously. Therefore, the responses to each odor were the average of at least six measurements. All experiments were done at room temperature $\left(22-25^{\circ} \mathrm{C}\right)$.

Odorant solutions. All odorant solutions were prepared as $0.5 \mathrm{M}$ stocks in DMSO and were then diluted with water to the concentration for EOG recording, $10^{-3} \mathrm{M}$. Those with low volatilities were additionally prepared at $10^{-2} \mathrm{M}$. At $10^{-3} \mathrm{M}$, the DMSO concentration in solution was $0.2 \%(\mathrm{~V} / \mathrm{V})$. Responses to DMSO alone were the same as to clean air $(<0.5 \mathrm{mV})$. Three milliliters of the odorant solution were placed in a $10 \mathrm{ml}$ glass test tube and capped with a silicon stopper. The concentration of volatile odorant in the $7-\mathrm{ml}^{2}$ airspace was allowed to equilibrate for more than 1 hour. All solutions were used within four hours. Two 18-gauge needles provided the input and output ports for the odorant- containing vapor above the solution. For stimulation, a $100-\mathrm{ms}$ pulse of the odorant vapor at 9 psi was injected into the continuous stream of humidified air. The pulse was controlled by a Picospritzer solenoid controlled valve (General Valve, Fairfield, New Jersey). The odorant stimulus pathway was cleaned by air between each stimulus presentation. The minimum interval between two adjacent stimuli was one minute. All odorant chemicals were purchased from Sigma-Aldrich (Saint Louis, Missouri), except citronellal, tetrahydrocitral, 7 methyl octanal, 2,5,7-trimethyl-2-octenal and 3-methyl-4(-3-methyl-1-cyclohexyl) propanal.

The precise concentration of odor that reaches the sensory endings cannot be determined, but is estimated to be between one and two orders of magnitude lower than the solution concentration ${ }^{5}$. In measurements of single-cell activity, obtained by calcium imaging, concentrations of octanal as low as $300 \mathrm{nM}$ produced a response. More importantly, comparing the responses of a set of agonists at the same concentration (30 $\mu \mathrm{M}$ ), the profile of activity was the same as found in the EOG recordings.

Data analysis. Because of inter-animal variability in the responses to the large number of odors used, we adopted a normalization strategy to facilitate comparisons. All responses within an individual animal were normalized to the response to a standard odor, amyl acetate. The peak amplitude of the amyl acetate response was always given the value of unity, and all other responses were represented as a percentage of the amyl acetate response. Amyl acetate was chosen because it typically gives a large, robust and repeatable response. The amyl acetate responses varied from $0.8 \mathrm{mV}$ to $12 \mathrm{mV}$ over the entire sample size. The same procedures were used on data from uninfected, Ad-I7-infected and control-infected animals. To account for the temporal variability over the course of a two- to threehour experiment, the amyl acetate standard was delivered on every sixth trial, and intervening responses were normalized to the average of the preceding and following amyl acetate responses. In general, no more than 10 
different odors (including octanal) were tried in a single epithelium, and were applied in the same order and number as used with controls. For a particular set of odors, the order of application was varied between animals, and when testing compounds with large responses, these were given after testing odors with low responses. To obtain the infected minus control value, we subtracted the average control value from the average Ad-I7infected responses obtained in EOG recordings. The statistical significance of the data was evaluated with an unpaired $t$-test.

$\mathrm{Ca}^{2+}$ imaging recordings. Single olfactory neurons were isolated from infected epithelia as follows. Briefly, GFP-positive epithelia from infected animals were dissected out in oxygenated divalent-free rat Ringer's (145 mM NaCl, $5.6 \mathrm{mM} \mathrm{KCl}, 10 \mathrm{mM}$ HEPES, $10 \mathrm{mM}$ glucose, $4 \mathrm{mM}$ EGTA, pH 7.4). The tissue was then incubated at $37^{\circ} \mathrm{C}$ for $45 \mathrm{~min}$ in $5 \mathrm{ml}$ of divalent-free Ringer containing $5 \mathrm{mg} / \mathrm{ml}$ bovine serum albumin (Sigma, St Louis, Missouri), $0.5 \mathrm{mg} / \mathrm{ml}$ collagenase, $22 \mathrm{U} / \mathrm{ml}$ dispase (Gibco BRL, Grand Island, New York) and $50 \mu \mathrm{g} / \mathrm{ml}$ deoxyribonuclease II (Sigma). The tissue was then transferred to a normal rat Ringer's (138 mM NaCl, $5 \mathrm{mM} \mathrm{KCl}, 1 \mathrm{mM} \mathrm{CaCl}, 1.5 \mathrm{mM} \mathrm{MgCl}_{2}, 10 \mathrm{mM}$ HEPES, $10 \mathrm{mM}$ D-glucose, $\mathrm{pH}$ 7.4), and the cells were dissociated by tapping the tube containing the tissue. Cells $(400 \mu \mathrm{l})$ were plated onto Concanavalin A $(10 \mathrm{mg} / \mathrm{ml})$-coated glass coverslips placed in $35 \mathrm{~mm}$ Petri dishes and allowed to settle. After $20 \mathrm{~min}, 2 \mathrm{ml}$ of culture medium was added to each dish, and the dishes were placed at $37^{\circ} \mathrm{C}$ in a $\mathrm{CO}_{2}$ incubator for at least 1 hour. The culture medium consisted of DMEM/F12 (Gibco BRL) supplemented with $10 \% \mathrm{FBS}, 100 \mu \mathrm{M}$ ascorbic acid, $1 \times$ insulin-transferrin-selenium-X (Gibco BRL), $2 \mathrm{mM}$ glutamine, $100 \mathrm{U} / \mathrm{ml}$ penicillin and $100 \mu \mathrm{g} / \mathrm{ml}$ streptomycin (Gibco BRL).

Calcium imaging recordings were done as described ${ }^{27}$. Cells were loaded with $5 \mu \mathrm{M}$ Fura-2/AM plus pluronic acid F127 (80 $\mu \mathrm{g} / \mathrm{ml}$; Molecular Probes, Eugene, Oregon) in rat Ringer's at room temperature $\left(22-25^{\circ} \mathrm{C}\right)$ in the dark for $45 \mathrm{~min}$. Imaging was done at room temperature on an inverted fluorescence microscope (IMT-2, Olympus, Tokyo, Japan) equipped with a SIT camera (C2400-08, Hamamatsu Photonics, Hamamatsu, Japan) connected to a frame grabber (LG-3, Scion, Frederick, Maryland) in a Macintosh computer. Imaging was controlled by the NIH Image 1.62 software operating a shutter (Uniblitz, Vincent Associates, Rochester, New York) to prevent photobleaching. Customized macros were written for shutter control and time-lapse imaging. Recordings were made at $380 \mathrm{~nm}$ excitation and $510 \mathrm{~nm}$ emission. Images were taken every four seconds, and three frames were averaged. The recording chamber was continuously perfused with oxygenated Ringer by means of a peristaltic pump. Odorant solutions (in rat Ringer's) were applied through syringes connected to the perfusion system with a manifold. Odors were applied for eight seconds in enough volume to completely replace the solution in the chamber $(200 \mu \mathrm{l})$. Data is shown as the fractional change in fluorescent light intensity: $\Delta \mathrm{F} / \mathrm{F}_{0}$ or $\left(\mathrm{F}-\mathrm{F}_{0}\right) / \mathrm{F}_{0}$, where $\mathrm{F}$ is the fluorescent light intensity at each point, and $\mathrm{F}_{0}$ is the value of emitted fluorescent light before the stimulus application (baseline) ${ }^{27}$.

Molecular modeling. Calculations were done using the MM2* Force Field default parameters as set up in Macromodel v6.5 (Schrodinger, Jersey City, New Jersey) for the energy minimization as well as for the Monte Carlo simulation ${ }^{28}$. Five thousand structures were minimized for each compound, for the conformational search. A conformational search of all compounds resulted in a number of possible conformations. The predicted lowest energy conformer from each of the searches was used in the overlay and to determine the molecular length of the molecules. The molecular length of all the molecules was calculated using atom center distances. Overlays were done using the carbonyl oxygen atom and carbon atoms $\mathrm{C} 1$ and $\mathrm{C} 4$ in a rigid body fit. Within a $10 \mathrm{~kJ}$ per mole range, the number of conformations obtained for each of the compounds in Fig. 4 were as follows: octanal, 56; trans-2-octenal, 35; trans, trans-2,4octadienal, 7; citral, 35; tetrahydrocitral, 181; citronellal, 182; 3-methyl4-(4-methylhexyl)-propanal, 26; 2,5,7-trimethyl-2-octenal, 33; 2,4-dimethyl-2,6-heptadienal, 17; trans-2-cis-6-nonadienal, 94.

\section{ACKNOWLEDGEMENTS}

We thank T. Loggia and C. Zhang for their assistance with the virus preparation and animal infection, $\mathrm{X}$. Zhang for his help with the $\mathrm{Ca}^{2+}$ imaging experiments, C. Margot and Firminch for chemicals and discussion, B. Schilling and Givaudan-Roure (Vernier, Switzerland) for chemicals, Takasago (Kanagawa, Japan) for neral and geranial, and P. Mombaerts for comments. This work was supported by the Whitehall and McKnight Foundations and the NIDCD.

\section{RECEIVED 18 July; ACCEPTED 24 OCTOBER 2000}

1. Beets, M. G. J. Structure-Activity Relationship in Human Chemoreception (Applied Science, London, 1978).

2. Mori, K., Nagao, H. \& Yoshihara, Y. The olfactory bulb: coding and processing of odor molecule information. Science 286, 711-715 (1999).

3. Buck, L. \& Axel, R. A novel multigene family may encode odorant receptors: a molecular basis for odor recognition. Cell 65, 175-187 (1991).

4. Mombaerts, P. Seven transmembrane proteins as odorant and chemosensory receptors. Science 286, 707-711 (1999).

5. Zhao, H. et al. Functional expression of a mammalian odorant receptor. Science 279, 327-242 (1998).

6. Malnic, B., Hirono, J., Sato, T. \& Buck, L. B. Combinatorial receptor codes for odors. Cell 96, 713-723 (1999).

7. Rubin, B. D. \& Katz, L. C. Optical imaging of odorant representations in the mammalian olfactory bulb. Neuron 23, 499-511 (1999).

8. Duchamp-Viret, P., Chaput, M. A. \& Duchamp, A. Odor response properties of rat olfactory receptor neurons. Science 284, 2171-2174 (1999).

9. Bozza, T. C. \& Kauer, J. S. Odorant response properties of convergent olfactory receptor neurons. J. Neurosci. 18, 4560-4569 (1998).

10. Touhara, K. et al. Functional identification and reconstitution of an odorant receptor in single olfactory neurons. Proc. Natl. Acad. Sci. USA 96, 4040-4045 (1999).

11. Krautwurst, D., Yau, K. W. \& Reed, R. R. Identification of ligands for olfactory receptors by functional expression of a receptor library. Cell 95, 917-926 (1998).

12. Wetzel, C. H. et al. Specificity and sensitivity of a human olfactory receptor functionally expressed in human embryonic kidney 293 cells and Xenopus laevis oocytes. J. Neurosci. 19, 7426-7433 (1999).

13. Marriott, D. P., Dougall, I. G., Meghani, P., Liu, Y. J. \& Flower, D. R. Lead generation using pharmacophore mapping and three-dimensional database searching: application to muscarinic $\mathrm{M}(3)$ receptor antagonists. J. Med. Chem. 42, 3210-3216 (1999).

14. Bikker, J. A., Trumpp-Kallmeyer, S. \& Humblet, C. G-protein coupled receptors: models, mutagenesis, and drug design. J. Med. Chem. 41, 2911-2927 (1998)

15. Zhao, H., Otaki, J. M. \& Firestein, S. Adenovirus-mediated gene transfer in olfactory neurons in vivo. J. Neurobiol. 30, 521-530 (1996).

16. Mackay-Sim, A. \& Kesteven, S. Topographic patterns of responsiveness to odorants in the rat olfactory epithelium. J. Neurophysiol. 71, 150-160 (1994).

17. Ottoson, D. Handbook of Sensory Physiology (Olfaction) (Springer, Berlin, 1971).

18. Serizawa, S. et al. Mutually exclusive expression of odorant receptor transgenes. Nat. Neurosci. 3, 687-693 (2000).

19. Shepard, G. M. in The Synaptic Organization of the Brain (ed. Shepard, G. M.) 159-204 (Oxford Univ. Press, New York, 1998).

20. Duchamp-Viret, P. \& Duchamp, A. Odor processing in the frog olfactory system. Prog. Neurobiol. 53, 561-602 (1997).

21. Firestein, S., Picco, C. \& Menini, A. The relation between stimulus and response in olfactory receptor cells of the tiger salamander. J. Physiol. (Lond.) 468, 1-10 (1993).

22. Singer, M. S. Analysis of the molecular basis for octanal interactions in the expressed rat I7 olfactory receptor. Chem. Senses 25, 155-165 (2000).

23. Pilpel, Y. \& Lancet, D. The variable and conserved interfaces of modeled olfactory receptor proteins. Protein Sci. 8, 969-977 (1999).

24. Hashimoto, M. et al. A neural cell-type-specific expression system using recombinant adenovirus vectors. Hum. Gene Ther. 7, 149-158 (1996).

25. Kanegae, Y., Makimura, M. \& Saito, I. A simple and efficient method for purification of infectious recombinant adenovirus. Jpn. J. Med. Sci. Biol. 47 157-166 (1994).

26. Niwa, H., Yamamura, K. \& Miyazaki, J. Efficient selection for highexpression transfectants with a novel eukaryotic vector. Gene 108, 193-200 (1991).

27. Yuste, R., Lanni, F. \& Konnerth, A. (eds.). Imaging Neurons: A Laboratory Manual (Cold Spring Harbor Laboratory Press, New York, 2000).

28. Mohamadi, F. et al. MacroModel-an integrated software system for modeling organic and bioorganic molecules using molecular mechanics. J. Comput. Chem. 11, 440-467 (1990). 